\title{
Population Frequency
}

National Cancer Institute

\section{Source}

National Cancer Institute. Population Frequency. NCI Thesaurus. Code C45373.

A description of the relative rate of occurrence of specific alleles, traits, or other factors within a population of organisms. 